\title{
Magnetic resonance imaging analysis of screw in-type lateral anchor pull-out in large to massive rotator cuff repair in patients older than 60 years
}

\author{
Sang-Yoon Lee, Young-Min Noh \\ Department of Orthopaedic Surgery, Dong-A University Hospital, Dong-A University College of Medicine, Busan, Korea
}

\begin{abstract}
Background: This study was performed to identify the incidence of screw in-type lateral anchor pull-out in patients older than 60 years who underwent rotator cuff repair for large to massive rotator cuff tear (RCT).

Methods: We reviewed 25 patients over 60 who were diagnosed with large to massive RCT and underwent arthroscopic rotator cuff repair in our hospital from March 2017 to February 2021. Preoperative tear size (anterior to posterior, medial to lateral) was measured via preoperative magnetic resonance imaging (MRI). All 25 patients underwent MRI scanning on postoperative day 1 and at 3 months after surgery. The change of anchor position was measured in axial views on MRI images postoperative day 1 and 3 months after surgery. And it was statistically compared according to bone mineral density (BMD), sex, and number of lateral anchors.

Results: Two MRIs (postoperative day 1 and 3 months) in 25 patients were compared. Anchor pull-out occurred in six patients during 3 months (6.7\%), and the mean pull-out length difference was $1.56 \mathrm{~mm}$ (range, $0.16-2.58 \mathrm{~mm}$ ). There was no significant difference in the number of pull-out anchors, degree of pull-out difference by comparing BMD (A, BMD $\leq-2.5 ; \mathrm{B}, \mathrm{BMD}>-2.5)$, sex, or number of anchors used in each surgery ( $C$, two anchors; $D$, three anchors) ( $\mathrm{p}>0.05)$.

Conclusions: Pull-out of screw in-type anchors was rarely observed and the mean pull-out length difference was negligibly small in our study. The screw in-type lateral anchor seems to be a decent option without concern of anchor pull-out even in elderly patients.
\end{abstract}

Keywords: Lateral anchor; Magnetic resonance imaging; Rotator cuff; Pull-out

\section{INTRODUCTION}

A rotator cuff tear is the leading cause of shoulder pain and dysfunction, and the prevalence of rotator cuff tears increases with age, with rates as high as $30 \%$ to $54 \%$ in adults over the age of 60 years [1-3]. There are several repair techniques for a rotator cuff tear, and suture bridge repair is preferred for its mechanical superiority to single-row repair [4-7]. Although advances of anchor and suture techniques have contributed to successful surgical outcomes, anchor loosening or pull-out of the medial or lateral anchor point will affect the integrity of the repaired cuff [8-10]. Even in elderly patients over 60 years of age, rotator cuff repair is

Received: June 6, $2021 \quad$ Revised: August 11, $2021 \quad$ Accepted: August 11, 2021

Correspondence to: Young-Min Noh

Department of Orthopaedic Surgery, Dong-A University Hospital, Dong-A University College of Medicine, 32 Daesingongwon-ro, Seo-gu, Busan 49201, Korea

Tel: +82-51-240-5166, Fax: +82-51-254-6757, E-mail: thugdoc@naver.com, ORCID: https://orcid.org/0000-0001-7149-7526

\section{Financial support: None.}

Conflict of interest: None.

Copyright@C 2022 Korean Shoulder and Elbow Society.

This is an Open Access article distributed under the terms of the Creative Commons Attribution Non-Commercial License (http://creativecommons.org/licenses/by-nc/4.0/) which permits unrestricted non-commercial use, distribution, and reproduction in any medium, provided the original work is properly cited. 
a very common procedure, and anchor pull-out is a concern because the possibility in patients with poor bone quality is relatively high $[11,12]$.

Suture bridge repair and use of lateral anchors is the most common technique in arthroscopic rotator cuff repair in the past decade. Although lateral anchors have been reported to have good biomechanical pull-out strength $[13,14]$, there are clinical concerns of screw loosening or pull-out of the lateral anchor in elderly patients, since the lateral portion of the greater tuberosity (GT) is composed of sparse cancellous bone [15,16]. Furthermore, bone mineral density (BMD) does not reflect accurately bone quality of the lateral GT because it is calculated most often in the hip and spine areas. Even in elderly patients with good $\mathrm{BMD}$, when inserting a screw in-type anchor, a loose fit during screw purchase is not uncommon, indicating questionable anchor stability.

There are some reports of lateral anchor pull-out. Tsiouri and Mok [17] reported a lateral row anchor that failed at 2 weeks after surgery, and Martinel and Bonnevialle [18] detected anchor pull-out by ultrasound at 6 weeks after surgery. However, as far as we know, there are no studies evaluating lateral anchor pullout based on analysis of serial follow-up radiographic images. This study was performed to identify the incidence of screw intype lateral anchor pull-out in patients older than 60 years who underwent rotator cuff repair for large and massive rotator cuff tears. We hypothesized that the degree of pull-out is correlated with variables of BMD, sex, and number of anchors, which can affect bone quality and pull-out strength.

\section{METHODS}

\section{Ethical Statement}

This study was approved by the Institutional Review Board of Dong-A University Hospital (IRB No. 21-122). Owing to the retrospective design, the requirement for informed consent was waived.

\section{Patient and Imaging Selection}

This study reviewed 243 patients who were diagnosed with rotator cuff tear and underwent arthroscopic rotator cuff repair in Dong-A University Hospital from March 2017 to February 2021. Of 243 patients, 43 receiving screw in-type lateral anchor were followed via magnetic resonance imaging (MRI) on postoperative day 1 and at 3 months after surgery. Of these, five patients were younger than 60 years, six had small to medium-sized rotator cuff tears, three had no BMD tests, and four underwent postoperative MRI scans in other hospitals; all were excluded from the analysis. A total of 25 patients was included in the current study and retrospectively reviewed. Demographic data were collected using electronic medical records from our hospital (Table 1). MRI (Discovery 750; GE Healthcare, Chicago, IL, USA) scans were performed at 3.0 Tesla (Water, Inphase, Outphase, short T1 inversion recovery) phases in our hospital. The change of lateral row anchor position was measured in serial axial views on MRI images. Images were interpreted using a standard picture archiving and communication system (PACS) workstation (Centricity, GE Healthcare). To measure the pull-out length of the anchor, we set a new parameter, the distance of anchor protrusion from the outermost surface of the cortical bone. First, the insertion point was located for the lateral anchor in the serial axial views. Second, a straight line was drawn along the outermost line of the cortex at the insertion point. Then, a line parallel to the first line was drawn at the most protruding part of the anchor. Finally, the vertical distance between the two parallel straight lines was measured, and the longest distance in the serial axial views was selected (Fig. 1). Both authors performed these measurements. Inter-observer variability was assessed using the intraclass correla-

Table 1. Demographics data

\begin{tabular}{lc}
\hline Variable & Patient $(\mathrm{n}=25)$ \\
\hline Age $(\mathrm{yr})$ & $67.32 \pm 5.01(60$ to 77$)$ \\
Sex (male:female) & $10: 15$ \\
Follow-up period (mo) & $10.48 \pm 5.56(3$ to 22$)$ \\
BMD & $-1.39 \pm 1.07(-3.15$ to -0.10$)$ \\
Tear size $(\mathrm{mm})$ & \\
$\quad$ ML & $40.52 \pm 10.35(30.18$ to 58.00$)$ \\
AP & $37.99 \pm 7.61(29.56$ to 50.82$)$ \\
\hline
\end{tabular}

Values are presented as mean \pm standard deviation (range).

BMD: bone mineral density, ML: medial to lateral, AP: anterior to posterior.
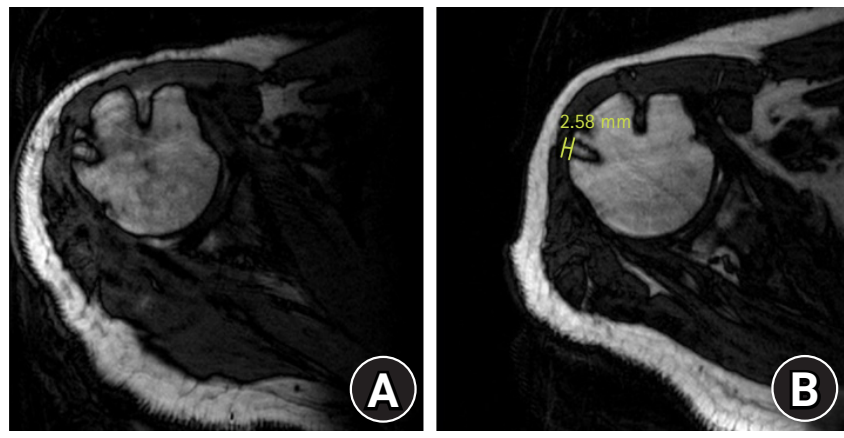

Fig. 1. Magnetic resonance imaging images on (A) postoperative day 1 and (B) 3 months after surgery with the same view (Outphase axial T2 IDEAL). The length of lateral row anchor pull-out was assessed by measuring the distance anchors pulled out perpendicular to the outer lateral cortex. 
tion coefficient, which was 0.802 (95\% confidence interval, $0.763-0.887$ ) for the proposed parameter.

\section{Surgical Technique}

All surgeries were performed by a single surgeon (YMN) with patients in the beach chair position under general anesthesia with an interscalene block. Diagnostic examination of the glenohumeral intraarticular region was performed using a $30^{\circ}$ arthroscope via the posterior portal. After moving the scope to the subacromial space, bursectomy and subacromial decompression were performed via anterior and lateral portals. The size and tear configuration of the rotator cuff were identified through lateral and posterolateral portals.

After biceps tenotomy or tenodesis and routine acromioplasty, the rotator cuff tissue was mobilized to its fullest extent, and careful assessment was made as to which repair configuration would yield the most complete repair at the lowest tension. Margin convergence and medialization were conducted if necessary to lateralize the free margin of the rotator cuff tear and to minimize strain at the repair site. Triple-loaded medial anchors (2.3 mm Iconix; Stryker, Kalamazoo, MI, USA) were placed at the articular margin of cartilage according to tear size, and six strands of FiberWire (Arthrex, Naples, FL, USA) from each anchor were passed just lateral to the musculotendinous margin of the torn rotator cuff using suture hook with shuttle relay technique. Knot tying at the medial anchor was performed with an SMC sliding knot. After five to seven strands of FiberWire were loaded in the lateral anchor through the islet, two or three 5.5-mm biocomposite lateral row screw in-type anchors $(5.5 \mathrm{~mm}$ BioComposite Swivelock, Arthrex) were inserted just lateral to the bicipital groove, at the lateral and posterior aspects of GT after FiberWire tightening. After confirming the repaired tendon and anchors to be well maintained in the subacromial and glenohumeral joints, all portals were closed layer by layer.

A postoperative rehabilitation protocol was followed for all patients, starting with passive shoulder range of motion after shoulder immobilization for 6 weeks using a shoulder abduction brace. At 12 weeks postoperatively, progressive muscle-strengthening exercises were started.

\section{Statistical Analysis}

Data are presented as mean \pm standard deviation. Categorical variables were analyzed using chi-square test or Fisher exact test, and continuous variables were analyzed using independent t-test or Mann-Whitney U-test. Parameters were evaluated using the Wilcoxon signed-rank test. Statistical analyses were performed using IBM SPSS ver. 19 (IBM Corp., Armonk, NY, USA). The level of significance was set at $\mathrm{p}<0.05$.

\section{RESULTS}

A total of 25 patients with large to massive rotator cuff tears who underwent MRI at postoperative day 1 and 3 months after surgery was examined. The mean age of the patients was $67.32 \pm 5.01$ years (range, 60-77 years). There were 10 men with a mean age of $65.30 \pm 5.06$ years and 15 women with a mean age of $68.67 \pm 4.65$ years. The mean BMD score was $-1.39 \pm 1.07$ (range, -3.15 to -0.10 ). The mean tear size in the coronal and sagittal planes was $40.52 \pm 10.35 \mathrm{~mm}$ (range, 30.18-58.00 mm) and $37.99 \pm 7.61 \mathrm{~mm}$ (range, 29.56-50.82 mm), respectively (Table 1). Among 25 patients compared on two consecutive MRI scans (postoperative day 1 and 3 months), anchor pull-out occurred in six $(6.7 \%)$, and the mean pull-out length was $1.56 \mathrm{~mm}(0.16-$ 2.58).

Of the 25 patients, six (24\%) had osteoporosis (BMD $\leq-2.5$; A group), and 19 (76\%) had normal bone density or osteopenia (BMD > -2.5; B group). Our method was not able to analyze correlation between BMD and pull-out number, but the number of these occurrences was $0(0 \%)$ in the A group and six (31.58\%) in the $\mathrm{B}$ group (Table 2 ).

The mean pull-out length was $0.79 \pm 1.10 \mathrm{~mm}$ in the male group and $0.10 \pm 0.33 \mathrm{~mm}$ in the female group. The number of cases in the male group with pull-out was four (40\%), and two (13.33\%) were observed in the female group. The pull-out length and number were not statistically different between the two groups ( $\mathrm{p}>0.05)$ (Table 3 ).

Of the 25 patients, 14 (56\%) used two lateral anchors (C group)

Table 2. Comparison of age, sex, tear size, re-tear, and accompanying pull-out length and number between two groups

\begin{tabular}{lccc}
\hline Variable & A group $^{*}$ & B group $^{\dagger}$ & p-value \\
\hline No. of patients & $6(24)$ & $19(76)$ & - \\
Age (yr) & $70.17 \pm 5.27$ & $66.42 \pm 4.71$ & 0.133 \\
Sex & & & 0.051 \\
$\quad$ Male & 0 & 10 & \\
$\quad$ Female & 6 & 9 & \\
Tear size (mm) & & & \\
$\quad$ ML & $37.44 \pm 7.42$ & $41.50 \pm 11.10$ & 0.949 \\
$\quad$ AP & $34.01 \pm 4.21$ & $39.25 \pm 8.08$ & 0.227 \\
Re-tear & 1 & 5 & 1.000 \\
Pull-out length (mm) & $0.00 \pm 0.00$ & $0.49 \pm 0.89$ & 0.126 \\
Pull-out no. & 0 & 6 & 0.278 \\
\hline
\end{tabular}

Values are presented as number (\%) or mean \pm standard deviation. ML: medial to lateral, AP: anterior to posterior.

${ }^{\star}$ Bone mineral density $(\mathrm{BMD}) \leq-2.5 ;{ }^{\dagger} \mathrm{BMD}>-2.5$. 
Table 3. Comparison of age, tear size, BMD, re-tear, and accompanying pull-out length and number between sex groups

\begin{tabular}{lccc}
\hline Variable & Male & Female & p-value \\
\hline No. of patients & $10(40)$ & $15(60)$ & - \\
Age $(\mathrm{yr})$ & $65.30 \pm 5.06$ & $68.67 \pm 4.65$ & 0.106 \\
Tear size $(\mathrm{mm})$ & & & \\
$\quad$ ML & $43.54 \pm 11.66$ & $38.51 \pm 9.23$ & 0.360 \\
$\quad$ AP & $41.69 \pm 8.31$ & $35.52 \pm 6.22$ & 0.052 \\
BMD & $-0.68 \pm 0.70$ & $-1.86 \pm 1.02$ & 0.005 \\
Re-tear & 4 & 2 & 0.175 \\
Pull-out length (mm) & $0.79 \pm 1.10$ & $0.10 \pm 0.33$ & 0.089 \\
Pull-out no. & 4 & 2 & 0.175 \\
\hline
\end{tabular}

Values are presented as number (\%) or mean \pm standard deviation. BMD: bone mineral density, ML: medial to lateral, AP: anterior to posterior.

and $11(44 \%)$ used three lateral anchors (D group). The average pull-out length was $0.15 \pm 0.51 \mathrm{~mm}$ in the $\mathrm{C}$ group and $0.66 \pm 1.02$ $\mathrm{mm}$ in the $\mathrm{D}$ group. The number of cases with pull-out was two (14.29\%) in the C group and four (36.36\%) in the D group. The pull-out length and pull-out number did not differ significantly between the two groups ( $\mathrm{p}>0.05)$ (Table 4 ).

\section{DISCUSSION}

In this study, we focused on the incidence of screw in-type lateral anchor pull-out recognized by serial MRI during the first 3 months after large to massive rotator cuff repair in patients older than 60 years. The results of this study indicate that BMD, age, and lateral anchor number do not yield a significant difference in outcome.

There are previous studies that have shown the pull-out of anchors following rotator cuff repair [19-22]. Kaar et al. [19] reported the complications of metallic suture anchors and showed that, while the use of metallic suture anchors in the shoulder is common and useful, there are remarkable risks if the anchors are placed improperly. In a study by Djurasovic et al. [20], suture anchor loosening occurred in eight patients, all of whom had to undergo repeated repair of the rotator cuff tendon to bone attachment. Moreover, several studies addressed early anchor pullout. Dezaly et al. [21] reported a prevalence of 3.1\% early anchor pull-out in 127 patients, while Benson et al. [22] detected early suture anchor pull-out at a rate of $2.4 \%$ in 269 patients. Dezaly et al. [21] found that the benefit of repair was especially clear in intermediate tears, and Benson et al. [22] reported that anchor pull-out risk increases with tear size. There are some previous studies regarding the pull-out of lateral row anchor $[17,18]$. Tsiouri and Mok [17] reported a lateral row anchor that failed at
Table 4. Comparison of age, sex, tear size, re-tear, and accompanying pull-out length and number between two groups

\begin{tabular}{lccc}
\hline Variable & C group $^{*}$ & D group $^{\dagger}$ & p-value \\
\hline No. of patient & $14(56)$ & $11(44)$ & - \\
Age $(\mathrm{yr})$ & $67.57 \pm 4.29$ & $67.00 \pm 6.00$ & 0.546 \\
Sex & & & 0.241 \\
$\quad$ Male & 4 & 6 & \\
$\quad$ Female & 10 & 5 & \\
Tear size (mm) & & & \\
$\quad$ ML & $35.02 \pm 8.23$ & $47.52 \pm 8.52$ & 0.003 \\
$\quad$ AP & $34.11 \pm 5.54$ & $42.93 \pm 7.16$ & 0.003 \\
Re-tear & 4 & 2 & 0.661 \\
Pull-out length (mm) & $0.15 \pm 0.51$ & $0.66 \pm 1.02$ & 0.165 \\
Pull-out no. & 2 & 4 & 0.350 \\
\hline
\end{tabular}

Values are presented as number (\%) or mean \pm standard deviation. ML: medial to lateral, AP: anterior to posterior.

${ }^{\star}$ Two lateral anchors; ${ }^{\dagger}$ Three lateral anchors.

2 weeks after surgery and recommend caution when placing lateral anchors on the GT with suspected osteopenia. Martinel and Bonnevialle [18] performed ultrasonography to detect anchor pull-out at 6 weeks after surgery, and three patients showed a mean pull-out distance of $8.3 \mathrm{~mm}$. Existing studies did not serialize imaging tests through MRI and have short follow-up. However, in our study, we compared the MRI results at postoperative day 1 and at 3 months after surgery and analyzed the degree of pull-out. We chose this short-term follow-up based on the assumption that most anchor pull-out would occur in the early postoperative period. We thought that well maintained anchors at 3 months after surgery would have achieved coaptation with the bone, leaving little chance of later pull-out. As a result, the screw in-type lateral anchor did not pull-out easily in the first 3 months regardless of bone quality. Anchor pull-out was observed in six patients (6.7\%), and the average length of anchor pull-out was very small $(1.56 \mathrm{~mm})$.

Some of the factors affecting anchor stability are cuff repair technique, cuff tear size, BMD, sex, age, anchor number, and anchor location $[4,6,13,15]$. Osteoporotic changes in the proximal humerus can decrease the stability of anchor fixation and cause problems in rotator cuff repair [20,23]. The site for re-attachment of tendons in rotator cuff repair varies by patient, and surgeons determine the appropriate location depending on cuff tear size, degree of tendon retraction, and degree of firm fixation after anchor insertion [24,25]. Since the risk of anchor pull-out increases with decreasing BMD at anchor placement, BMD values of anchor insertion sites are important and vary depending on the specific location of the proximal humerus [12,16]. However, these characteristics are not significantly related to our results. 
There might be selection bias in our study, and unlike previous studies, our results suggest that BMD is not a significant factor related to pull-out length ( $\mathrm{p}>0.05)$.

It can be predicted that anchor pull-out will be higher in female patients due to poor bone quality because of lower BMD than males according to a previous study [12], but there was no significant difference in number or degree of anchor pull-out between male and female patients in our study ( $p>0.05$ ) (Table 3).

In rotator cuff repair, larger cuff tear sizes can affect pull-out by transmitting greater tension to the lateral row anchor. Kulwick et al. [26] suggested that load was transferred through the entire portion of the tendon, as well as the medial row and lateral row anchors in suture bridge repair. Accordingly, the larger is the tear size, the greater is the tension on the anchor, and the higher is the tendency for the anchor to pull-out. However, this was not observed at a significant level in our study $(\mathrm{p}>0.05)$.

The GT can be divided into three portions of anterior, middle, and posterior (Fig. 2), and BMD values vary within the GT $[12,15]$. Kirchhoff et al. [27] reported that BMD was higher in the posterior portion of the GT, and Tingart et al. [12,16] showed highest trabecular BMD values in the posteromedial region and highest cortical BMD values at the mid-medial aspect. In our study, mild anchor pull-out was observed in the anterior area in three cases, middle in two cases, and posterior in one case. The degree of pull-out according to location of the anchor relative to the GT was not confirmed statistically due to the insufficient number of samples (Table 5). Not a single anchor was pulled out by more than $5 \mathrm{~mm}$, indicating little chance of complete anchor pull-out. This outcome seems to result from a decrease in lateral anchor tension as medial row anchors initially bear a load from rotator cuff tension with an increase in pull-out strength due to the high pitch of suture anchors.

We chose patients with large to massive rotator cuff tear older than 60 years for the following reasons. First, MRI scanning of postoperative day one was performed only in such patients. Sec- ond, evaluation was thought to be needed because the larger is the size of the rotator cuff tear, the greater will be the retracted tension stress at each anchor, and the greater is the susceptibility to pull-out of the lateral anchor, especially in elderly patients with poor bone quality $[16,28]$. Third, evaluation was conducted considering both BMD and age, since we have clinical experience that firm fixation cannot be achieved when anchors are placed loosely at the insertion site despite normal BMD values in patients older 60 years. In addition, the reason for limiting the study to screw in-type anchors was that we thought bone quality would be more affected than with push in-type anchors attached

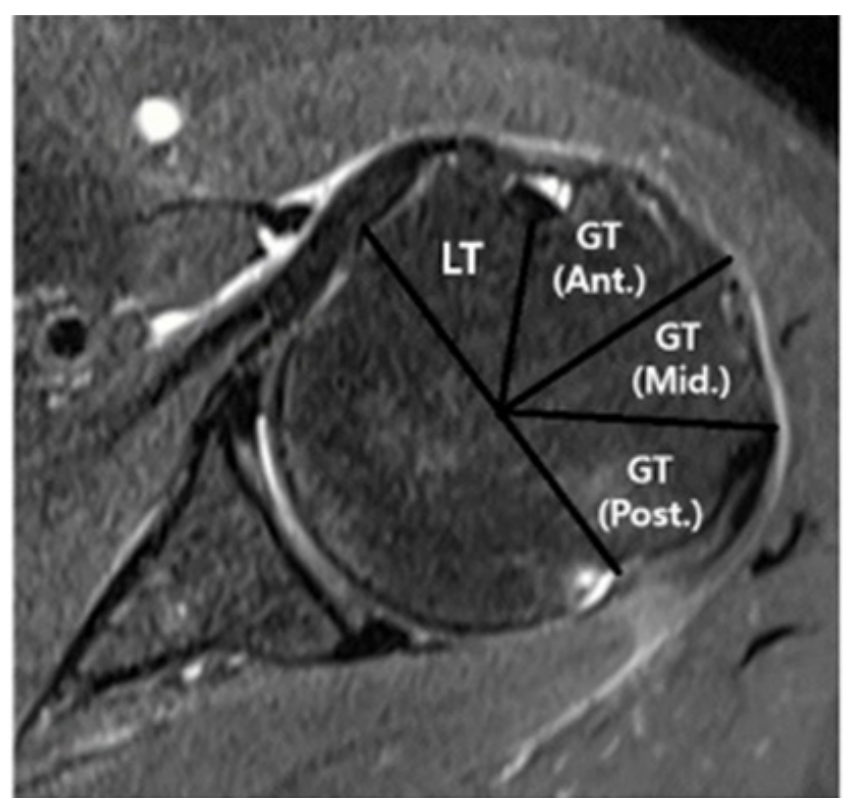

Fig. 2. The greater tuberosity (GT) can be divided into three portions of anterior (Ant), middle (Mid), and posterior (Post). In our study, a total of six patients demonstrated anchor pull-out. The mean age of these patients was $67.17 \pm 5.04$ years. Mild anchor pull-out was observed in the Ant area in three cases, Mid in two cases, and Post in one case. The average pull-out length according to location was 1.50 $\mathrm{mm}$ in the Ant, $1.79 \mathrm{~mm}$ in the Mid, and $1.29 \mathrm{~mm}$ in the Post area. LT: lesser tuberosity.

Table 5. Anchor pull-out case information

\begin{tabular}{|c|c|c|c|c|c|c|}
\hline \multirow{2}{*}{ Variable } & \multicolumn{6}{|c|}{ Patient no. } \\
\hline & 1 & 2 & 3 & 4 & 5 & 6 \\
\hline Age(yr) & 62 & 73 & 64 & 65 & 65 & 74 \\
\hline Sex & $\mathrm{F}$ & $\mathrm{M}$ & $\mathrm{F}$ & M & $\mathrm{M}$ & $\mathrm{M}$ \\
\hline $\mathrm{BMD}$ & -0.1 & -0.3 & -0.3 & -0.1 & -1.5 & -1.7 \\
\hline No. of anchors & 2 & 3 & 3 & 2 & 3 & 3 \\
\hline Pull-out length (mm) & 0.16 & 0.99 & 1.29 & 1.90 & 2.43 & 2.58 \\
\hline No. of tendons involved & 2 & 3 & 2 & 2 & 3 & 3 \\
\hline Pull-out location & Anterior & Middle & Posterior & Anterior & Anterior & Middle \\
\hline
\end{tabular}

BMD: bone mineral density. 
to the cortex, and we assumed study homogeneity with use of one type of anchor.

This study had several limitations. First, the most significant drawback of this study is that the sample size was too small. However, since the evaluation was limited to large and massive rotator cuff tear in patients over 60 years and limited to patients who underwent MRI at postoperative day 1 and at 3 months after surgery, the patient number of 25 is not insufficient. Second, the small number of groups with osteoporosis (BMD $\leq-2.5$ ) was not sufficient for a simple comparison. However, as mentioned in the text, it is not possible to evaluate the bone quality of the proximal humerus with simple BMD. The reason for this study was that, although BMD score was not bad, firm fixation could not be achieved in many cases when we placed the lateral anchor in the cancellous portion of the GT in elderly patients. This study is meaningful for its restriction of patients to those older than 60 years. Finally, the mean follow-up period was limited to only 3 months after surgery. However, as most anchor pull-out occurs in the early postoperative period, meaningful clinical importance is maintained with this time frame.

Screw in-type lateral anchor pull-out was observed in six cases (24\%), and the length of pull-out was negligibly small $(1.56 \mathrm{~mm})$ in first 3 months after surgery. Detailed comparisons were made according to BMD, sex, and number of anchors, but there was no statistically significant difference in number of pulled out anchors, degree of pull-out difference of lateral anchor, or re-tear rate $(p>0.05)$. As a result, the screw in-type lateral anchor seems to be a suitable option without concern of anchor pull-out even in elderly patients ( $>60$ years).

\section{ORCID}

Sang-Yoon Lee

Young-Min Noh

https://orcid.org/0000-0003-1424-0040

https://orcid.org/0000-0001-7149-7526

\section{REFERENCES}

1. Lehman C, Cuomo F, Kummer FJ, Zuckerman JD. The incidence of full thickness rotator cuff tears in a large cadaveric population. Bull Hosp Jt Dis 1995;54:30-1.

2. Milgrom C, Schaffler M, Gilbert S, van Holsbeeck M. Rotator-cuff changes in asymptomatic adults: the effect of age, hand dominance and gender. J Bone Joint Surg Br 1995;77:296-8.

3. Sher JS, Uribe JW, Posada A, Murphy BJ, Zlatkin MB. Abnormal findings on magnetic resonance images of asymptomatic shoulders. J Bone Joint Surg Am 1995;77:10-5.

4. Thangarajah T, Lo IK, Sabo MT. Rotator cuff repair techniques: current concepts. J Clin Orthop Trauma 2021;17:149-56.

5. Mihata T, Watanabe C, Fukunishi K, et al. Functional and structural outcomes of single-row versus double-row versus combined double-row and suture-bridge repair for rotator cuff tears. Am J Sports Med 2011;39:2091-8.

6. Cho NS, Yi JW, Lee BG, Rhee YG. Retear patterns after arthroscopic rotator cuff repair: single-row versus suture bridge technique. Am J Sports Med 2010;38:664-71.

7. Dines JS, Bedi A, ElAttrache NS, Dines DM. Single-row versus double-row rotator cuff repair: techniques and outcomes. J Am Acad Orthop Surg 2010;18:83-93.

8. Kim SJ, Choi YR, Jung M, Lee W, Chun YM. Arthroscopic repair of anterosuperior massive rotator cuff tears: does repair integrity affect outcomes. Am J Sports Med 2017;45:1762-8.

9. Kim KC, Shin HD, Lee WY, Han SC. Repair integrity and functional outcome after arthroscopic rotator cuff repair: double-row versus suture-bridge technique. Am J Sports Med 2012;40:294-9.

10. DeFranco MJ, Bershadsky B, Ciccone J, Yum JK, Iannotti JP. Functional outcome of arthroscopic rotator cuff repairs: a correlation of anatomic and clinical results. J Shoulder Elbow Surg 2007;16:759-65.

11. Jo YH, Lee KH, Kim SJ, Kim J, Lee BG. National trends in surgery for rotator cuff disease in Korea. J Korean Med Sci 2017; 32:357-64.

12. Tingart MJ, Apreleva M, Lehtinen J, Zurakowski D, Warner JJ. Anchor design and bone mineral density affect the pull-out strength of suture anchors in rotator cuff repair: which anchors are best to use in patients with low bone quality. Am J Sports Med 2004;32:1466-73.

13. Acan AE, Hapa O, Horoz L, Kara A, Havıtçıoğlu H. Effects of a lateral row anchor position on the suture holding strength of a double-row knotless fixation in rotator cuff repair. Eklem Hastalik Cerrahisi 2018;29:46-51.

14. Lafosse L, Brozska R, Toussaint B, Gobezie R. The outcome and structural integrity of arthroscopic rotator cuff repair with use of the double-row suture anchor technique. J Bone Joint Surg Am 2007;89:1533-41.

15. Sakamoto $\mathrm{Y}$, Kido A, Inoue K, et al. In vivo microstructural analysis of the humeral greater tuberosity in patients with rotator cuff tears using multidetector row computed tomography. BMC Musculoskelet Disord 2014;15:351.

16. Tingart MJ, Apreleva M, Zurakowski D, Warner JJ. Pullout strength of suture anchors used in rotator cuff repair. J Bone Joint Surg Am 2003;85:2190-8.

17. Tsiouri C, Mok DH. Early pullout of lateral row knotless anchor in rotator cuff repair. Int J Shoulder Surg 2009;3:63-5. 
18. Martinel V, Bonnevialle N. Contribution of postoperative ultrasound to early detection of anchor pullout after rotator cuff tendon repair: report of 3 cases. Orthop Traumatol Surg Res 2020; 106:229-34.

19. Kaar TK, Schenck RC Jr, Wirth MA, Rockwood CA Jr. Complications of metallic suture anchors in shoulder surgery: a report of 8 cases. Arthroscopy 2001;17:31-7.

20. Djurasovic M, Marra G, Arroyo JS, Pollock RG, Flatow EL, Bigliani LU. Revision rotator cuff repair: factors influencing results. J Bone Joint Surg Am 2001;83:1849-55.

21. Dezaly C, Sirveaux F, Philippe R, et al. Arthroscopic treatment of rotator cuff tear in the over-60s: repair is preferable to isolated acromioplasty-tenotomy in the short term. Orthop Traumatol Surg Res 2011;(6 Suppl):S125-30.

22. Benson EC, MacDermid JC, Drosdowech DS, Athwal GS. The incidence of early metallic suture anchor pullout after arthroscopic rotator cuff repair. Arthroscopy 2010;26:310-5.

23. Barber FA, Feder SM, Burkhart SS, Ahrens J. The relationship of suture anchor failure and bone density to proximal humerus location: a cadaveric study. Arthroscopy 1997;13:340-5.
24. Ticker JB, Warner JJ. Single-tendon tears of the rotator cuff. Evaluation and treatment of subscapularis tears and principles of treatment for supraspinatus tears. Orthop Clin North Am 1997;28:99-116.

25. Burkhart SS, Diaz Pagàn JL, Wirth MA, Athanasiou KA. Cyclic loading of anchor-based rotator cuff repairs: confirmation of the tension overload phenomenon and comparison of suture anchor fixation with transosseous fixation. Arthroscopy 1997; 13:720-4.

26. Kulwicki KJ, Kwon YW, Kummer FJ. Suture anchor loading after rotator cuff repair: effects of an additional lateral row. J Shoulder Elbow Surg 2010;19:81-5.

27. Kirchhoff C, Braunstein V, Milz S, et al. Assessment of bone quality within the tuberosities of the osteoporotic humeral head: relevance for anchor positioning in rotator cuff repair. Am J Sports Med 2010;38:564-9.

28. Davidson PA, Rivenburgh DW. Rotator cuff repair tension as a determinant of functional outcome. J Shoulder Elbow Surg 2000;9:502-6. 